\title{
Functional Results of Arthroscopic Treatment in Patients With Femoroacetabular and Subspine Impingement Diagnosed With a 3-Dimensional Dynamic Study
}

\author{
Bernardo Aguilera-Bohórquez, M.D., Salvador Ramirez, M.D., and Erika Cantor, M.Sc.
}

\begin{abstract}
Purpose: To describe the functional results of arthroscopic treatment in patients with femoroacetabular impingement (FAI) and subspine impingement (SSI) evaluated with a 3-dimensional (3D) dynamic study. Methods: This was a retrospective observational study of patients with a diagnosis of FAI and SSI, evaluated with a 3D dynamic computed tomography scan with Move Forward software, who underwent hip arthroscopy between February 2015 and December 2017. Measurements of the alpha angle, femoral anteversion, acetabular anteversion, lateral center-edge angle, and Tönnis angle were extracted from the 3D dynamic study. Functionality was evaluated using the Western Ontario McMaster Universities Osteoarthritis Index before and 12 months after surgery. Results: We analyzed 22 hips in 17 patients (9 female and 8 male patients) with an average age of $34.6 \pm 14.3$ years. Of the 22 hips, 15 had cam morphology, 6 had mixed morphology, and 1 had pincer morphology. Of the hips, 11 had a type I spine, 10 had type II, and 1 had type III. The average alpha angle, Tönnis angle, femoral anteversion, and acetabular anteversion were $61.9^{\circ} \pm 11.1^{\circ}, 2.5^{\circ} \pm$ $6.4^{\circ}, 8.8^{\circ} \pm 6.8^{\circ}$, and $15.1^{\circ} \pm 7.1^{\circ}$, respectively. The median lateral center-edge angle was $38.1^{\circ}$ (interquartile range, $\left.32.6^{\circ}-43.5^{\circ}\right)$. At 1 -year follow-up, a decrease in the Western Ontario McMaster Universities Osteoarthritis Index score $(P=.001)$ and an increase in the flexion angle $(P<.001)$ were observed. No cases needed posterior surgical revision because of persistent pain. Conclusions: Arthroscopic treatment provides symptom relief and good functional results in patients with FAI and SSI. Level of Evidence: Level IV, therapeutic case series.
\end{abstract}

$\mathbf{F}$ emoroacetabular impingement (FAI) is one of the main causes of hip pain in young adults. ${ }^{1}$ Recently, there has been particular interest in studying the extraarticular causes of impingement that could contribute to the symptomatology of hip pain and decreased range

From Orthopedics and Traumatology, Hip Preservation Unit, Institute of Osteoarticular Diseases (B.A-B.), Arthroscopic Hip Preservation Surgery, Pontificia Universidad Javeriana de Cali (S.R.), and Research Institute (E.C.), Centro Médico Imbanaco, Cali, Colombia; and Universidad de Valparaiso (E.C.), Valparaiso, Chile.

The authors report that they have no conflicts of interest in the authorship and publication of this article. Full ICMJE author disclosure forms are available for this article online, as supplementary material.

Received June 6, 2019; accepted October 27, 2019.

Address correspondence to Bernardo Aguilera-Bohórquez, M.D., Carrera 38 A No. 5A-100, Torre A, Office 305, Hip Preservation Unit, Institute of Osteoarticular Diseases, Centro Médico Imbanaco, Cali, Colombia. E-mail: baguilera@imbanaco.com.co

(C) 2019 THE AUTHORS. Published by Elsevier Inc. on behalf of the Arthroscopy Association of North America. This is an open access article under the CC BY-NC-ND license (http://creativecommons.org/licenses/by-nc-nd/4.0/). 2666-061X/19713

https://doi.org/10.1016/j.asmr.2019.10.007 of motion. Among the group of extra-articular impingement pathologies, subspine impingement (SSI) is characterized by abnormal contact between the femoral neck and the anterior inferior iliac spine (AIIS). ${ }^{2}$ The frequency of SSI in conjunction with FAI has been described as between $23.7 \%$ and $32.0 \%$ in patients with hip pain. ${ }^{3,4}$

Although there is not a specific validated test to make the diagnosis of SSI, the presence of pain during maximal flexion is considered suggestive of SSI. ${ }^{5}$ Underdiagnosis of SSI may result in residual impingement, even after arthroscopic management of FAI. $^{6}$ Currently, there are complementary studies such as computed tomography (CT) with 3-dimensional (3D) dynamic reconstruction that can guide us toward a specific treatment, avoiding negative and unexpected results in the postoperative period. ${ }^{7}$

Arthroscopic treatment of FAI has shown good results in patients with hip pain, ${ }^{8,9}$ and its use has extended to extra-articular causes such as SSI and ischiofemoral impingement among others. However, the number of publications that have described the clinical results of 
arthroscopic treatment of cases with a diagnosis of extra-articular impingement is low compared with the number of publications on FAI. The purpose of this study was to describe the functional results of arthroscopic treatment in patients with FAI and SSI evaluated with a 3D dynamic study. Our hypothesis was that hip arthroscopy would be a safe treatment with optimal functional results in cases with FAI and SSI.

\section{Methods}

This was a retrospective observational study of patients with a diagnosis of FAI and SSI who underwent hip arthroscopy between February 1, 2015, and December 31, 2017. The inclusion criteria were (1) patients who had preoperative evidence of FAI and SSI on a 3D dynamic study, (2) patients who had groin pain with a decreased range of flexion and who had pain during maximal hip flexion and/or pain on palpation of the AIIS on physical examination, and (3) patients who had undergone surgical treatment of FAI and SSI during the same surgical session. Patients with previous hip surgery were excluded. This study was approved by the institutional review board (Centro Médico Imbanaco Review Board) and was conducted in accordance with the principles of the Declaration of Helsinki.

Our institutional hip arthroscopy registry was reviewed to identify patients with a 3D dynamic study. Only cases that met the inclusion and exclusion criteria were reviewed and analyzed. Patients were evaluated through a physical examination and CT scan with 3D dynamic reconstruction and were treated with at least 3 months of conservative treatment. All cases had evidence of FAI morphology and abnormal contact between the AIIS and femoral neck on the 3D dynamic study, as shown in Figure 1. All images were assessed as described in previously published research. ${ }^{3}$

\section{Three-Dimensional Dynamic Reconstruction}

All images were obtained with CT scans (Brilliance CT 6 Slice; Philips Healthcare, Cleveland, OH) using a standardized protocol in a single institution. Pelvis and hip CT scans with knee cuts were performed using 2-mm-thick slices. The 3D reconstruction and dynamic study were performed using Clinical Graphics software (Move Forward; Zimmer Biomet, Miami, FL). This software creates segmentations and subsequent 3D models of the femoroacetabular morphology using an active shape-modeling technique ${ }^{10}$ to simulate the range of motion of the femoroacetabular joint based on the recommendations of the International Society of Biomechanics $^{11}$ and the equidistant method described by Puls et al. ${ }^{12}$

\section{Surgical Technique for AllS Decompression}

Arthroscopic AIIS decompression and surgical treatment of FAI were performed during the same session by a single orthopaedic surgeon (B.A-B), specializing in hip preservation. The arthroscope used was a Storz Power LED 175 unit (Karl Storz Endoskope, Tuttlingen, Germany) with $30^{\circ}$ and $70^{\circ}$ lens, with a Stryker flow-control arthroscopy pump (Kalamazoo, MI), a hand-controlled vaporization system (VAPR; DePuy, Raynham, MA), and a shaver device system (Karl Storz Endoskope). Hip arthroscopy was performed with the patient under regional and general anesthesia and placed in the supine position on an orthopaedic traction table, with the use of a perineal post. The hip was reached through the anterolateral, anterior, and accessory distal arthroscopic portals. The anterior portal was placed slightly more medial than usual to facilitate access to the AIIS. To achieve better visualization of the acetabular rim and the AIIS, tenosuspension of the reflective portion of the tendon of the rectus anterior muscle was performed. ${ }^{13}$ The hip was placed in $30^{\circ}$ of flexion to undergo an extended inferomedial capsulotomy. The tendon of the iliopsoas muscle was used as a point of reference to locate the AIIS, which was decompressed with a $5.5-\mathrm{mm}$ drill (Fig 2). Dynamic tests were performed to evaluate if osseous resection of the AIIS was sufficient according to the technique described by Locks et al. ${ }^{14}$ In all hips, an osteochondroplasty of the femoral head-neck junction was performed with the goal of increasing the femoral offset and decreasing the possibility of abnormal contact with the AIIS. Figure 3 shows the AIIS before and after arthroscopic decompression.

\section{Data Collection}

The AIIS morphology was classified according to Hetsroni et al. ${ }^{15}$ Type I was defined by a smooth ilium wall between the AIIS and the acetabular rim, type II was classified as the AIIS prominences extending from the AIIS to the acetabular rim, and type III was defined by the AIIS prominences extending distally to the anterosuperior acetabular rim.

Measurements of the alpha angle, femoral anteversion, acetabular anteversion, lateral center-edge angle, and Tönnis angle were gathered from the 3D dynamic study. Functionality was evaluated by the Western Ontario McMaster Universities Osteoarthritis Index (WOMAC) before and 12 months after the surgical procedure. The total score ranges from 0 to 100 , in which 0 represents the best functionality. A difference between the preoperative and postoperative scores of at least 11.5 points was considered the minimal clinically important difference. ${ }^{16}$ The presence of complications was recorded during data collection.

\section{Statistical Analysis}

Continuous variables were summarized as the mean \pm standard deviation or median and interquartile range (IQR). Qualitative variables were presented as absolute frequencies and proportions. Fitting to a 
Fig 1. Posterior (left), lateral (Middle), and anterior (Right) views of right hip by computed tomography with 3-dimensional reconstruction. (A) Morphology of evaluated hip. (B) Hip with FAI (femoroacetabular impingement) and subspine impingement morphology (Blue zone). The abnormal contact between the anterior inferior iliac spine (AIIS) and femoral neck is evidenced.

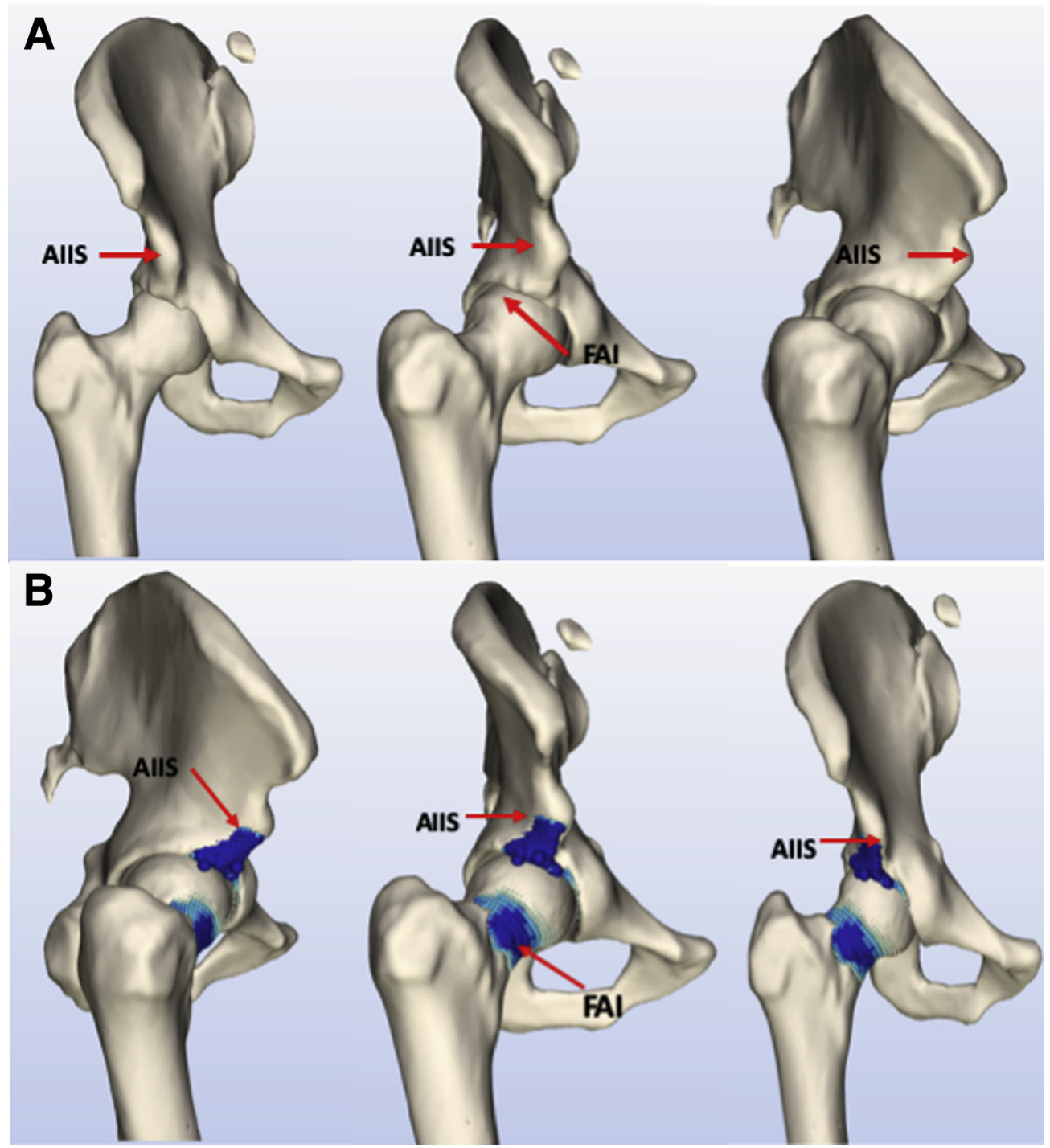

normal distribution was evaluated with the Shapiro Wilk test. The Wilcoxon test or paired $t$ test was used to evaluate differences before and after the procedure. $P<$ .05 was considered statistically significant. All analyses were conducted using the Statal3 program (StataCorp, College Station, TX).

\section{Results}

In this retrospective study, we initially identified 46 hips with abnormal contact between the AIIS and the femoral neck, as well as evidence of FAI morphology, on 3D dynamic CT scans. However, we included only 22 hips because they required surgical treatment for both pathologies given the evidence of clinical correlation between imaging studies and clinical findings and were treated in our center.

A total of 17 patients ( 9 female and 8 male patients) were analyzed, with an average age of $34.6 \pm 14.3$ years. The study included 11 right and 11 left hips. Of the 22 hips, 15 had cam morphology, 6 had mixed morphology, and 1 had pincer morphology. On the basis of the AIIS classification, 11 hips had a type I spine, 10 had type II, and 1 had type III. The average alpha angle, Tönnis angle, femoral anteversion, and acetabular anteversion were $61.9^{\circ} \pm 11.1^{\circ}, 2.5^{\circ} \pm 6.4^{\circ}$, $8.8^{\circ} \pm 6.8^{\circ}$, and $15.1^{\circ} \pm 7.1^{\circ}$, respectively. The median lateral center-edge angle was $38.1^{\circ}$ (IQR, 32.6 $-43.5^{\circ}$ ). The most frequent intraoperative findings were labral bruising and peripheral focal bruising of the AIIS (Table 1).

Improvement was noted in pain and function on the WOMAC 12 months after surgery, with a reduction in the scores. The minimal clinically important difference in the total score was reached in 14 of 17 patients $(82.3 \%)$. At 1 year after surgery, an average increase in hip flexion of $10^{\circ}$ occurred compared with the preoperative value $(P<.001)$; improvement in internal rotation was also observed (Table 2). Of the 22 hips that 


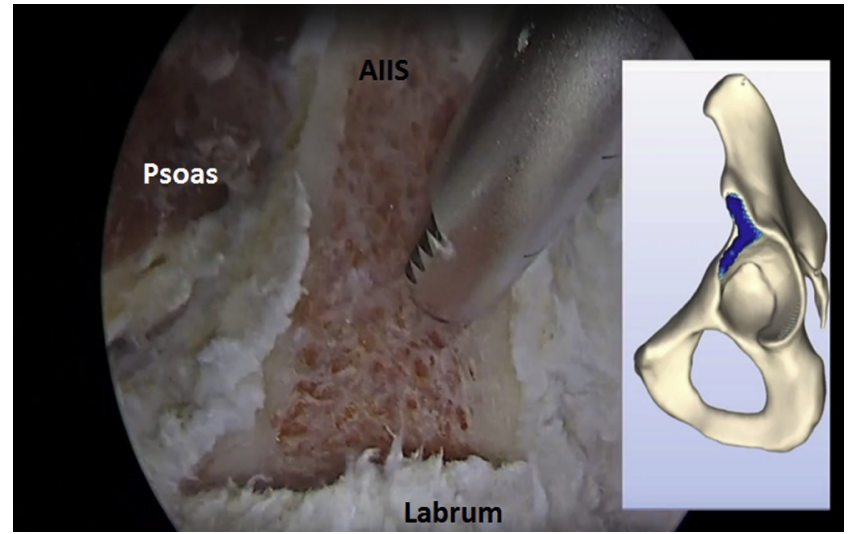

Fig 2. A dynamic study of the left hip shows abnormal contact between the pelvis (anterior inferior iliac spine [AIIS]) and femoral neck (blue zone). An endoscopic view shows the resection from the acetabular rim to the AIIS, suggesting that the findings of the 3-dimensional dynamic study are reproducible in clinical practice.

underwent intervention, $12(54.5 \%)$ had diminished femoral anteversion $\left(<10^{\circ}\right)$; of these, 7 had a type I spine and 5 had a type II spine. In cases with femoral retroversion, an average increase in flexion of $14^{\circ}$ was observed at 1-year follow-up $\left(106.8^{\circ} \pm 7.9^{\circ}\right.$ before surgery vs $120.0^{\circ} \pm 4.3^{\circ}$ after surgery, $P=.001$ ); this was similar to the finding reported in all cases. On the other hand, regarding internal rotation, a greater change in the postoperative period was found in cases with femoral retroversion (median, $20^{\circ}$ [IQR, 8.2 $25.5^{\circ}$ ] before surgery vs $29.0^{\circ}$ [IQR, $22.2^{\circ}-30.0^{\circ}$ ] after surgery, $P=.006$ ) when compared with all cases. At the end of the follow-up period, no complications were reported and no cases required reintervention because of residual pain.

\section{Discussion}

This study shows that arthroscopic surgery is a safe technique for the simultaneous treatment of FAI and SSI with good functional results and a low complication rate. These findings are similar to those of Souza et al., ${ }^{17}$ who described 2 cases of young male patients with a diagnosis of SSI and mixed-type FAI, with good results and full relief of symptoms. Arthroscopic treatment of SSI with optimal functional results has been described by other authors. ${ }^{2,15,18-21}$ Similarly, hip arthroscopy in the management of isolated FAI has shown clear benefits with a significant improvement in patient-reported outcomes after surgery. ${ }^{8}$

In our study, unlike in other studies, all patients with SSI underwent intervention with the certainty of an impact on the AIIS, evidenced by a dynamic 3D study. SSI is a pathology associated in many cases with FAI; for this reason, it may be underdiagnosed during clinical practice. The clinical results found by the WOMAC evidenced pain relief and functional improvement compared with the preoperative period, findings similar

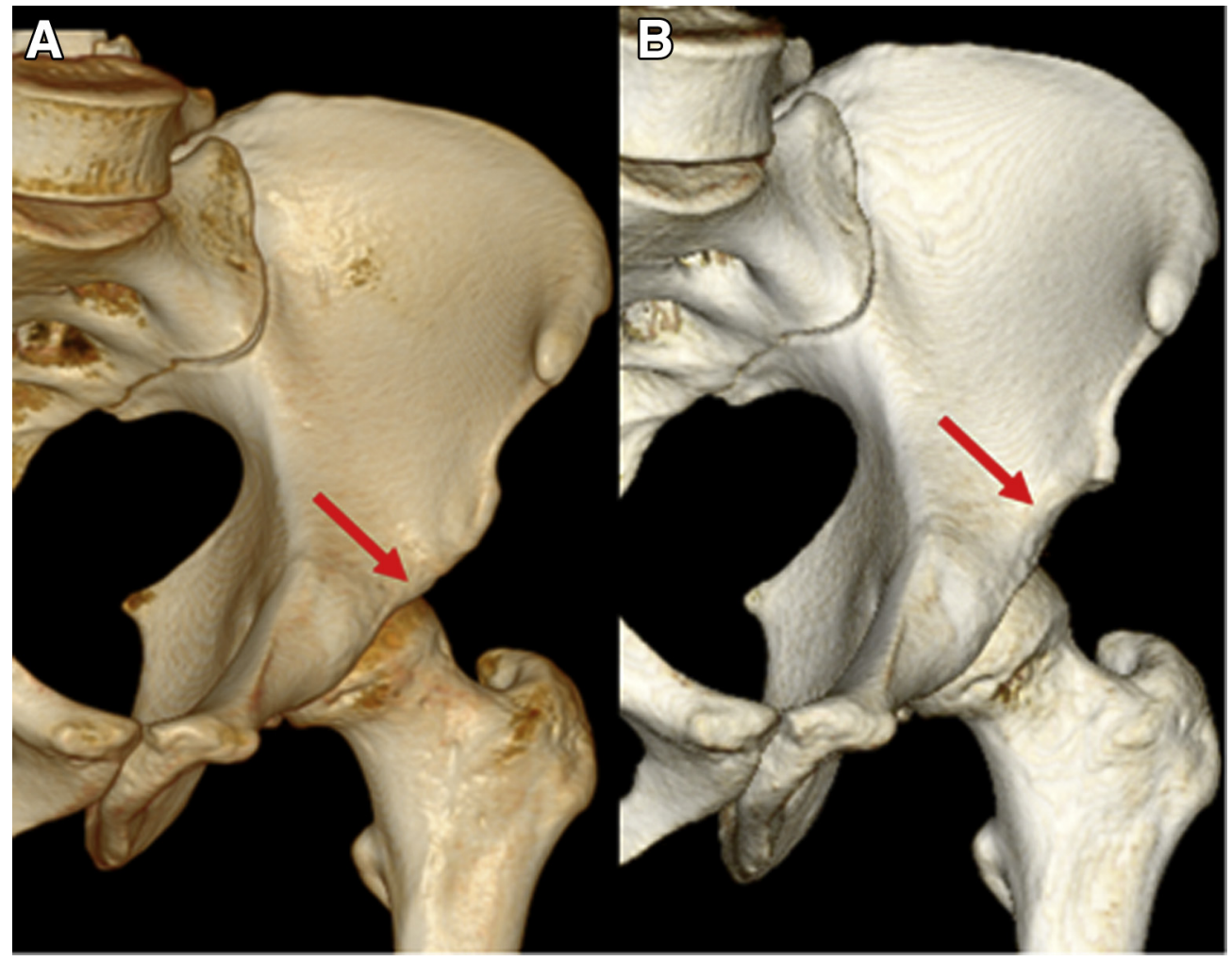

Fig 3. (A) Computed tomography scan of left hip before surgery. The arrow points to the anterior inferior iliac spine (AIIS). (B) Computed tomography scan of left hip after decompression of AIIS (arrow). 
Table 1. Diagnosis and Surgical Findings

\begin{tabular}{|c|c|}
\hline Variable & Hips $(\mathrm{N}=22), \mathrm{n}$ \\
\hline \multicolumn{2}{|l|}{ Comorbidities } \\
\hline Cam FAI & 15 \\
\hline Pincer FAI & 1 \\
\hline Mixed FAI & 6 \\
\hline \multicolumn{2}{|l|}{ Surgical findings } \\
\hline Lesion of labrum & 18 \\
\hline Focal AIIS synovitis & 3 \\
\hline Peripheral focal bruising of AIIS & 13 \\
\hline Focal ossification of anterior rim of acetabulum & 6 \\
\hline Chondral lesion & 5 \\
\hline
\end{tabular}

to those described using the modified Harris Hip Score. ${ }^{2,19-21}$ At the end of follow-up, none of the patients reported hip pain; this result suggests that resection of the AIIS in patients with clinical symptoms and imaging findings suggestive of FAI and SSI is an effective and reproducible treatment.

A reduction in flexion and internal rotation of the hip is the main clinical finding associated with a morphologic alteration of the AIIS. Hetsroni et al., ${ }^{15}$ in their study about morphologic classification of the AIIS, found a relation between the reduction in flexion and internal rotation and an increase in the prominence of the AIIS over the acetabular rim. Because of the sample size, our study did not corroborate these findings. However, after surgery, we found statistically significant improvement in the range of flexion and internal rotation. In our series, 1 of 22 hips $(4.5 \%)$ presented with heterotopic ossification associated with the procedure, which is considered the most frequent complication after hip arthroscopy. ${ }^{8}$

In this study, it was possible to evidence, using 3D dynamic CT scans, that abnormal contact does occur between an AIIS classified as type I and the femoral neck, which is similar to the findings reported by Zaltz et al. ${ }^{22}$ Half of the included hips had a type I AIIS; this finding supports the hypothesis that alterations of the AIIS morphology are not the main cause of SSI. In addition, a type II AIIS and a type III AIIS have been found in asymptomatic patients without associated hip comorbidities. ${ }^{19,23,24}$ SSI is one of the main causes of revision of primary hip arthroscopy, mainly because there are not specific tests for its identification and it is not a pathology widely recognized by hip surgeons. ${ }^{6}$ Larson et al. ${ }^{6}$ reported that up to $45.9 \%$ of patients with residual FAI who required reintervention had a prominent AIIS or had an extension toward the acetabular rim; in addition, decompression of the AIIS was associated with better functional results in their study.

In several reports, the diagnosis of SSI has been made mainly based on clinical symptoms, pain with maximal flexion, radiographs, and CT scans. During routine clinical practice, the diagnosis of SSI must be made based on clinical findings such as limitation of range of motion and pain elicited by maximal flexion and/or palpation over the AIIS. ${ }^{5,18}$ The use of CT helps to recognize other parameters such as the morphology of the AIIS; however, in many cases, these findings are not conclusive and it is recommended to perform dynamic studies that allow evaluation of the possible zones of conflict in the hip. ${ }^{7}$ The 3D dynamic study is a tool that allows one to perform a directed intervention, with the goal of avoiding the appearance of residual pain and reinterventions associated with the impingement of the AIIS. The causes of SSI are not attributable to a single specific etiology, and there is some controversy about the importance of the AIIS morphology as a determinant factor in the development of SSI. ${ }^{25}$ Aguilera-Bohorquez et al. $^{3}$ described that femoral retroversion of less than $8^{\circ}$ would increase the suggestion of SSI, even in hips with a type I AIIS. This finding was present in half of the hips in our study. Recently, Samim et al., ${ }^{4}$ in a cohort of 62 symptomatic patients who underwent arthroscopic treatment for FAI, reported that evidence of soft-tissue injuries and osseous findings on preoperative magnetic resonance imaging could be considered the main features associated with SSI.

\section{Limitations}

Among the limitations of this study were the following: (1) Patients had a 1-year follow-up period, which could be considered a limited follow-up for a

Table 2. Functional Results Before and After Arthroscopic Procedure

\begin{tabular}{|c|c|c|c|}
\hline Clinical Results & Preoperative & Postoperative & $P$ Value \\
\hline \multicolumn{4}{|l|}{ WOMAC score, median (IQR) } \\
\hline Pain & $10.0(7.7-13.5)$ & $3.0(0-5.2)$ & $.001^{*}$ \\
\hline Function & $11.5(5.0-27.2)$ & $4.0(0.7-10.2)$ & $.007^{*}$ \\
\hline Total & $31.0(25.2-42.0)$ & $10.0(2.5-18.2)$ & $.001^{*}$ \\
\hline Flexion, mean $\pm \mathrm{SD}$, degrees & $108.8 \pm 7.5$ & $119.6 \pm 4.8$ & $<.001^{*}$ \\
\hline Internal rotation, median (IQR), degrees & $24.5(18.7-29.2)$ & $30.0(25.7-32.5)$ & $<.001^{*}$ \\
\hline
\end{tabular}

IQR, interquartile range; SD, standard deviation; WOMAC, Western Ontario McMaster Universities Osteoarthritis Index.

*Statistically significant $(P<.05)$. 
surgical technique. (2) The lack of a comparison group does not allow us to determine if the functional results of simultaneous surgical treatment are better than those of isolated management of FAI or SSI. However, it is important to note that the number of published studies on combined management of FAI and SSI is low. (3) The use of the WOMAC does not allow a direct comparison with other studies in which the evaluation of functionality was performed with the modified Harris Hip Score. (4) Although all data were gathered from a prospective institutional registry and every image was reviewed by trained staff, this study has the disadvantages of a retrospective study because the clinical information was transcribed into the registry as part of routine care. (5) The findings of this study are considered limited because of the small sample size and short follow-up time.

\section{Conclusions}

Arthroscopic treatment provides symptom relief and good functional results in patients with FAI and SSI.

\section{Acknowledgment}

The authors appreciate the Research Institute of Centro Médico Imbanaco for its support during the development of this project.

\section{References}

1. Ganz R, Parvizi J, Beck M, Leunig M, Notzli H, Siebenrock KA. Femoroacetabular impingement: A cause for osteoarthritis of the hip. Clin Orthop Relat Res 2003;417: 112-120.

2. Hetsroni I, Larson CM, Dela Torre K, Zbeda RM, Magennis E, Kelly BT. Anterior inferior iliac spine deformity as an extra-articular source for hip impingement: A series of 10 patients treated with arthroscopic decompression. Arthroscopy 2012;28:1644-1653.

3. Aguilera-Bohorquez B, Brugiatti M, Coaquira R, Cantor E. Frequency of subspine impingement in patients with femoroacetabular impingement evaluated with a 3dimensional dynamic study. Arthroscopy 2019;35:91-96.

4. Samim M, Walter W, Gyftopoulos S, Poultsides L, Youm T. MRI assessment of subspine impingement: Features beyond the anterior inferior iliac spine morphology. Radiology 2019;293:412-421.

5. Poultsides LA, Bedi A, Kelly BT. An algorithmic approach to mechanical hip pain. HSS J 2012;8:213-224.

6. Larson CM, Giveans MR, Samuelson KM, Stone RM, Bedi A. Arthroscopic hip revision surgery for residual femoroacetabular impingement (FAI): Surgical outcomes compared with a matched cohort after primary arthroscopic FAI correction. Am J Sports Med 2014;42: 1785-1790.

7. Gupta AK, Abrams GD, Nho SJ. What's new in femoroacetabular impingement surgery: Will we be better in 2023? Sports Health 2014;6:162-170.
8. Minkara AA, Westermann RW, Rosneck J, Lynch TS. Systematic review and meta-analysis of outcomes after hip arthroscopy in femoroacetabular impingement. Am J Sports Med 2019;47:488-500.

9. Nwachukwu BU, Rebolledo BJ, McCormick F, Rosas S, Harris JD, Kelly BT. Arthroscopic versus open treatment of femoroacetabular impingement. Am J Sports Med 2015;44:1062-1068.

10. Roling MA, Visser MI, Oei EHG, Pilot P, Kleinrensink G-J, Bloem RM. A quantitative non-invasive assessment of femoroacetabular impingement with CT-based dynamic simulation-Cadaveric validation study. BMC Musculoskelet Disord 2015;16:50.

11. Wu G, Siegler S, Allard P, et al. ISB recommendation on definitions of joint coordinate system of various joints for the reporting of human joint motion-Part I: Ankle, hip, and spine. J Biomech 2002;35:543-548.

12. Puls M, Ecker TM, Tannast M, Steppacher SD, Siebenrock KA, Kowal JH. The equidistant method-A novel hip joint simulation algorithm for detection of femoroacetabular impingement. Comput Aided Surg 2010;15:75-82.

13. Aguilera-Bohorquez B, Gil E, Fonseca J, Fernandez M, Sánchez M. Tenosuspension of the reflected head of the rectus femoris in hip arthroscopy: Description of a portal and a surgical maneuver. Arthrosc Tech 2017;6: el015-e1019.

14. Locks R, Chahla J, Mitchell JJ, Soares E, Philippon MJ. Dynamic hip examination for assessment of impingement during hip arthroscopy. Arthrosc Tech 2016;5:e1367-e1372.

15. Hetsroni I, Poultsides L, Bedi A, Larson CM, Kelly BT. Anterior inferior iliac spine morphology correlates with hip range of motion: A classification system and dynamic model. Clin Orthop Relat Res 2013;471:2497-2503.

16. Harris JD, Brand JC, Cote MP, Faucett SC, Dhawan A. Research pearls: The significance of statistics and perils of pooling. Part 1: Clinical versus statistical significance. Arthroscopy 2017;33:1102-1112.

17. Souza BGSE, Cardoso RM, Loque RS, Monte LFR, Sabino JP, Oliveira VM. Mixed-type femoroacetabular impingement associated with subspine impingement: Recognizing the trifocal femoropelvic impingement. Rev Bras Ortop 2018;53:389-394.

18. Larson CM, Kelly BT, Stone RM. Making a case for anterior inferior iliac spine/subspine hip impingement: Three representative case reports and proposed concept. Arthroscopy $2011 ; 27: 1732-1737$.

19. Hapa O, Bedi A, Gursan O, et al. Anatomic footprint of the direct head of the rectus femoris origin: Cadaveric study and clinical series of hips after arthroscopic anterior inferior iliac spine/subspine decompression. Arthroscopy 2013;29:1932-1940.

20. Nawabi DH, Degen RM, Fields KG, Wentzel CS, Adeoye O, Kelly BT. Anterior inferior iliac spine morphology and outcomes of hip arthroscopy in soccer athletes: A comparison to nonkicking athletes. Arthroscopy 2017;33 4:758-765.

21. Nwachukwu BU, Chang B, Fields K, et al. Outcomes for arthroscopic treatment of anterior inferior iliac spine (subspine) hip impingement. Orthop J Sport Med 2017;5: 2325967117723109. 
22. Zaltz I, Kelly BT, Hetsroni I, Bedi A. The crossover sign overestimates acetabular retroversion. Clin Orthop Relat Res 2013;471:2463-2470.

23. Yoo JI, Ha YC, Lee HJ, Lee JY, Lee YK, Koo KH. No difference in prevalence of radiographic subspinal impingement of the hip between symptomatic and asymptomatic subjects. Knee Surg Sports Traumatol Arthrosc 2017;25: $1951-1957$.
24. Wong TT, Bloom MC, Kazam JK, Ahmed FS, Rasiej MJ. Anterior inferior iliac spine morphology: Comparison of symptomatic hips with femoroacetabular impingement and asymptomatic hips. AJR Am J Roentgenol 2019;212: 166-172.

25. Carton P, Filan D. Anterior inferior iliac spine (AIIS) and subspine hip impingement. Muscles Ligaments Tendons J 2016;6:324-336. 